\title{
A Nonlinear Parabolic Model in Processing of Medical Image
}

\author{
R. Aboulaich ${ }^{a}$, S. Boujena ${ }^{b 1}$ and E. El Guarmah ${ }^{a}$ \\ ${ }^{a}$ LERMA, Mohammadia Engineering School, Mohamed V University, Ibn Sina Str. \\ POB 765 Agdal, Rabat, Morocco \\ ${ }^{b}$ Mathematics and computing Department, Ain Chock Sciences Faculty, Km8 Route El Jadida \\ BOP 5366 Maârif, Casablanca
}

\begin{abstract}
The image's restoration is an essential step in medical imaging. Several Filters are developped to remove noise, the most interesting are filters who permits to denoise the image preserving semantically important structures. One class of recent adaptive denoising methods is the nonlinear Partial Differential Equations who knows currently a significant success. This work deals with mathematical study for a proposed nonlinear evolution partial differential equation for image processing. The existence and the uniqueness of the solution are established. Using a finite differences method we experiment the validity of the proposed model and we illustrate the efficiently of the method using some medical images. The Signal to Noise Ration (SNR) number is used to estimate the quality of the restored images.
\end{abstract}

Key words: nonlinear parabolic model, image processing, Hilbert space

AMS subject classification: 68U10, 94A08

\section{Introduction}

Restoration is a crucial step in image processing. Numerous algorithms have been proposed recently to tackle the problems of noise removal and image restoration in real images $([4,7,8,9,14$, 17]).

\footnotetext{
${ }^{1}$ Corresponding author. E-mail: boujena@yahoo.fr, s.boujena@fsac.ac.ma
} 
We are interested to restore the noisy image $u_{0}$ using the following nonlinear PDE problem

$$
\left\{\begin{array}{l}
\frac{\partial u}{\partial t}-\operatorname{div}[\mu(|\nabla u|) \nabla u]=0 \text { in } Q \\
u(x, t)=0 \quad \forall x \in \partial \Omega \quad \forall t \in[0, T], \\
u(x, 0)=u_{0} \quad \forall x \in \Omega .
\end{array}\right.
$$

Where $\Omega$ is an open bounded subset of $\mathbb{R}^{n}, n=2$ or $n=3$, with boundary $\partial \Omega, Q=\Omega \times[0, T]$ with some given $T>0$.

Many algorithms are proposed for image processing $[6,10,12,15,16]$. The existence and the uniqueness of a solution of the used PDE problems are in general difficult to establish, an approach using the regularizing kernel is proposed in several works, see [3, 4]. In [1] the authors prove the existence and the uniqueness solution in Orlicz space. In this work we establishe the existence and uniqueness in $H^{1}(\Omega)$ space, under suitable hypothesis on $\mu$.

The problem (1.1) is equivalent to the following variational problem:

Find $u \in V$ such that:

$$
\int_{\Omega} \frac{\partial u(t)}{\partial t} v d x+\int_{\Omega} \mu[|\nabla u(t)|] \nabla u(t) . \nabla v d x=0 .
$$

Notice that $\mathcal{V}=\mathcal{D}(\Omega), V=H_{0}^{1}(\Omega)$ is the adherence of $\mathcal{V}$ in $H^{1}(\Omega) . H_{0}^{1}(\Omega)$ will be provided with the scalar product $((u, v))=\int_{\Omega} \nabla u . \nabla v d x$ of associated norm $\|$. $\|$. We indicate by $H$ the adherence of $\mathcal{V}$ in $L^{2}(\Omega)$. The space $H$ is provided with the scalar product of $L^{2}(\Omega)$ defined by: $(u, v)=\int_{\Omega} u v d x$. The associated norm is noted by $|$.$| , see [13]. In the following we$ will establish the existence and the uniqueness of the weak solution of (1.1) under the following hypothesis, see [5, 11], on $\mu$ :

(1) $\mu: \mathbb{R}_{+} \rightarrow \mathbb{R}_{+}$

(2) $\mu$ is continuous function

(3) $\lim _{s \rightarrow+\infty}[\mu(s)]=\mu_{0}$ with $\mu_{0}>0$

(4) $\mu$ is differential continuous

(5) $s\left|\mu^{\prime}(s)\right| \leq \mu(s) \forall s \in \mathbb{R}_{+}$

The hypothesis $((1)-(3))$ involve that $\mu$ is bounded.

Notice that $\sup _{s \in \mathbb{R}_{+}} \mu(s)=a$ and $\inf _{s \in \mathbb{R}_{+}} \mu(s)=b$, with $a \geq 0$ and $b \geq 0$.

We denote by $A$ the operator defined by

$$
(A v, w)=\int_{\Omega} \mu(|\nabla v|) \nabla v \cdot \nabla w d x \quad \text { for } v, w \in V
$$


According the hypothesis on $\mu$ we have $A v \in V^{\prime}$ if $v \in V$, where $V^{\prime}=H^{-1}(\Omega)$ is the dual space of $V$. We prove, see [5], that $A$ is an operator monotone hemicontinuous, satisfying for all $u, v \in V$

$$
(A u-A v, u-v) \geq b\|u-v\|^{2}
$$

\section{Existence theorem}

Theorem 1. Let $u_{0} \in H$ and $\mu$ satisfying (1)-(5). Then there exists at least one weak solution $u$ of problem (1) such that $u \in L^{2}(0, T, V) \cap L^{\infty}(0, T, H)$.

If $b=0$ the existence takes place for any finish $T$.

If $b \neq 0$ the existence is global.

Proof. To show the existence, we use a Faedo-Galerkin method. We consider the spectral problem

$$
((w, v))=\lambda(w, v) \quad \forall v \in V .
$$

the injection of $V$ in $H$ is compact, the problem (2.1) admits a sequence of eigenvalues $\lambda_{j}$ associated of eigenvectors $w_{j}$ such that

$$
\left(\left(w_{j}, v\right)\right)=\lambda_{j}\left(w_{j}, v\right) \quad \forall v \in V .
$$

and $\left\{w_{j}\right\}_{j \in \mathbb{N}}$ is orthonormal in $H$ and orthogonal in $V$. We denote $u_{N}(t)$ the approximate solution of (1.1) defined by

$$
u_{N}(x, t)=u_{N}(t)(x) \in\left[w_{1}, \ldots, w_{N}\right] \quad u_{N}(x, t)=\sum_{j=1}^{N} C_{j}^{N}(t) w_{j}(x)
$$

We have then

$$
\left\{\begin{array}{r}
\left(u_{N}^{\prime}(t), w_{j}\right)+\left(\mu\left(\left|\nabla u_{N}(t)\right|\right) \nabla u_{N}(t), \nabla w_{j}\right)=0 \quad 1 \leq j \leq N, t \in[0, T] \\
\text { with } u_{N}(., 0)=u_{0 N}(.) \in\left[w_{1}, \ldots, w_{N}\right], \text { and } u_{0 N} \rightarrow u_{0} \text { in } V .
\end{array}\right.
$$

Each $C_{j}^{N}(t)$ verifies $\frac{d C_{j}^{N}(t)}{d t}=G_{j}\left(t, C_{1}^{N}(t), \ldots, C_{N}^{N}(t)\right)$, where $G_{j}$ is a continuous function, then by using the Cauchy-Péano theorem we deduce that there exists a local solution $u_{N}(t)$ of $(2.4)$ on $\left[0, T_{N}\right]$.

By multiplying (2.4) by $C_{j}^{N}(t)$ and by adding, we deduct that:

$$
\int_{\Omega} \frac{\partial u_{N}(t)}{\partial t} u_{N}(t) d x+\int_{\Omega} \mu\left[\left|\nabla u_{N}(t)\right|\right]\left(\nabla u_{N}(t)\right)^{2} d x=0
$$

Then

$$
\frac{1}{2} \frac{d}{d t}\left|u_{N}(t)\right|^{2}+\int_{\Omega} \mu\left[\left|\nabla u_{N}(t)\right|\right]\left(\nabla u_{N}(t)\right)^{2} d x=0
$$


case $1: b>0$.

As

$$
\int_{\Omega} \mu\left[\left|\nabla u_{N}(t)\right|\right]\left(\nabla u_{N}(t)\right)^{2} d x \geq b \int_{\Omega}\left(\nabla u_{N}(t)\right)^{2} d x
$$

From (2.5) we have

$$
\frac{1}{2} \frac{d}{d t}\left|u_{N}(t)\right|^{2}+b \int_{\Omega}\left(\nabla u_{N}(t)\right)^{2} d x \leq 0
$$

Then

$$
\frac{1}{2} \frac{d}{d t}\left|u_{N}(t)\right|^{2}+b\left\|u_{N}(t)\right\|^{2} \leq 0
$$

There exists thus a constant $C_{1}=\left|u_{0}\right|>0$ and a constant $C_{2}=\frac{\left|u_{0}\right|^{2}}{2 b}>0$ depending of $b$ and $u_{0}$ such that

$$
\left|u_{N}(t)\right| \leq C_{1} \quad \text { and } \quad \int_{0}^{t}\left\|u_{N}(\tau)\right\|^{2} d \tau \leq C_{2} \quad \forall t \in\left[0, T_{N}\right], \forall N \in \mathbb{N} .
$$

case $2: b=0$.

In (2.5), we will rewrite the second term in the form

$$
\int_{\Omega} \mu\left\{\nabla u_{N}(t)\right\}\left(\nabla u_{N}(t)\right)^{2} d x=\int_{\Omega}\left\{\mu\left[\nabla u_{N}(t)\right]-\mu_{0}\right\}\left(\nabla\left[u_{N}(t)\right]\right)^{2} d x+\mu_{0} \int_{\Omega}\left(\nabla u_{N}(t)\right)^{2} d x
$$

Where $\mu_{0}$ is given by (3). From (2.5) we have

$$
\left\{\begin{aligned}
\frac{1}{2} \frac{d}{d t}\left|u_{N}(t)\right|^{2}+\mu_{0}\left\|u_{N}(t)\right\|^{2} & =-\int_{\Omega}\left\{\mu\left[\nabla u_{N}(t)\right]-\mu_{0}\right\}\left(\nabla u_{N}(t)\right)^{2} d x \\
& \leq \int_{\Omega}\left|\mu\left[\nabla u_{N}(t)\right]-\mu_{0}\right|\left(\nabla u_{N}(t)\right)^{2} d x
\end{aligned}\right.
$$

Notice that $\mu_{1}(s)=\mu(s)-\mu_{0}$, and by (3), it was $\lim _{s \rightarrow+\infty} \mu_{1}(s)=0$ then for all $\varepsilon>0$, there exists $A>0$ so that for all $(x, t) \in \Omega \times\left[0, T_{N}\right]$

$$
\left|\mu_{1}\left\{\nabla u_{N}(x, t)\right\}\right|<\varepsilon \quad \text { if }\left|\nabla u_{N}(x, t)\right|>A
$$

Let $t \in\left[0, T_{N}\right]$ fixed, we consider the following sets

$$
\begin{gathered}
\Omega_{1}^{t}=\left\{x \in \Omega /\left|\nabla u_{N}(x, t)\right| \leq A\right\} \\
\Omega_{2}^{t}=\left\{x \in \Omega /\left|\nabla u_{N}(x, t)\right|>A\right\} \\
\Omega_{1}^{t} \cap \Omega_{2}^{t}=\emptyset \quad \Omega_{1}^{t} \cup \Omega_{2}^{t}=\Omega
\end{gathered}
$$


We have then:

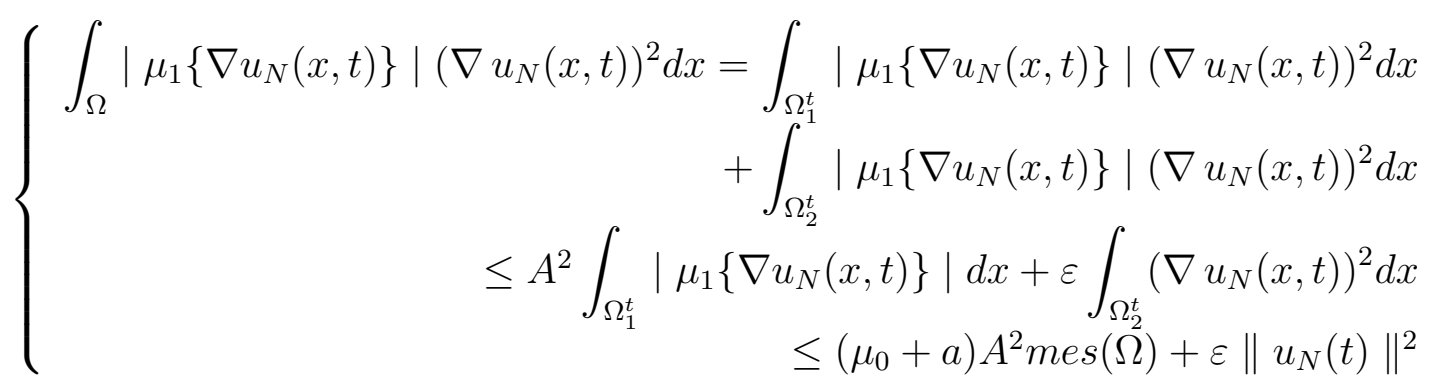

From (2.10) we have

$$
\frac{1}{2} \frac{d}{d t}\left|u_{N}(t)\right|^{2}+\mu_{0}\left\|u_{N}(t)\right\|^{2} \leq\left(\mu_{0}+a\right) A^{2} \operatorname{mes}(\Omega)+\varepsilon\left\|u_{N}(t)\right\|^{2}
$$

we take $\varepsilon=\frac{\mu_{0}}{2}$ and we set $\left(\mu_{0}+a\right) A^{2} \operatorname{mes}(\Omega)=C\left(\Omega, \mu_{0}, a\right)$, then

$$
\frac{1}{2} \frac{d}{d t}\left|u_{N}(t)\right|^{2}+\frac{\mu_{0}}{2}\left\|u_{N}(t)\right\|^{2} \leq C\left(\Omega, \mu_{0}, a\right)
$$

hence, by the same reasoning as the first case, we deduced that there exists a constant $C_{1}=\sqrt{2 C\left(\Omega, \mu_{0}, a\right)+\left|u_{0}\right|^{2}}>0$ and a constant $C_{2}=\frac{1}{\mu_{0}}\left(2 C\left(\Omega, \mu_{0}, a\right) T+\left|u_{0}\right|^{2}\right)>0$ depending of $\Omega, \mu_{0}, a, u_{0}$ and $T$ such that

$$
\left|u_{N}(t)\right| \leq C_{1} \quad \text { and } \int_{0}^{t}\left\|u_{N}(t)\right\|^{2} d t \leq C_{2} \quad \forall t \in\left[0, T_{N}\right], \forall N \in \mathbb{N}
$$

Thus in both cases there exists two constants $C_{1}>0$ and $C_{2}>0$ independent of $N$ such that

$$
\left\{\begin{array}{l}
\left|u_{N}(t)\right| \leq C_{1} \\
\int_{0}^{t}\left\|u_{N}(\tau)\right\|^{2} d \tau \leq C_{2}
\end{array} \quad \forall t \in\left[0, T_{N}\right], \forall N \in \mathbb{N}\right.
$$

Moreover $T_{N}=T$ for all $N \in \mathbb{N}$ and the problem (2.4) admits a unique solution

$u_{N} \in L^{2}(0, T, V) \cap L^{\infty}(0, T, H)$ on $[0, T]$ and according to the monotony and the hemicontinuity of the operator $A$ we deduce that the approximate solution $u_{N}$ of the problem (2.1) converges towards a weak solution $u$ of the problem $(1.1)$ (see [2, 5]).

Theorem 2. Under hypothesis of the theorem 1. with $b \neq 0$ we have

$$
u \in L^{2}(0,+\infty, V) \cap L^{\infty}(0,+\infty, H)
$$

and $\lim _{t \rightarrow+\infty}|u(t)|=0$. 
Proof. From the theorem 1., there exists $u \in L^{2}(0,+\infty, V) \cap L^{\infty}(0,+\infty, H)$. We have :

$$
\frac{1}{2} \frac{d}{d t}|u(t)|^{2}+\int_{\Omega} \mu[|\nabla u(t)|](\nabla u(t))^{2} d x=0,
$$

we deduce :

$$
\frac{1}{2} \frac{d}{d t}|u(t)|^{2}+b\|u(t)\|^{2} \leq 0
$$

Then

$$
\frac{1}{2} \frac{d}{d t}|u(t)|^{2}+\frac{b}{C(\Omega)^{2}}|u(t)|^{2} \leq 0
$$

where $C(\Omega)$ is a Poincare constant.

From (2.14) we deduce that $|u(t)|$ converge to 0 exponentially when $t \rightarrow+\infty$.

\section{Uniqueness Theorem}

Theorem 3. Under Hypothesis of the existence theorem, the weak solution u of the problem (1.1) is unique and $u^{\prime}=\frac{d u}{d t} \in L^{2}\left(0, T, V^{\prime}\right)$.

Proof. Let $u_{1}$ and $u_{2}$ two solutions of the problem (1.1), we have then for all $v \in V$

$$
\left(\frac{\partial u_{1}}{\partial t}(t)-\frac{\partial u_{2}}{\partial t}(t), v\right)+\left(A u_{1}(t)-A u_{2}(t), v\right)=0 .
$$

We take $u_{1}-u_{2}=w$ and $v=w(t)$ in (3.1), we have then from (1.4):

$$
\frac{1}{2} \frac{d}{d t}|w(t)|^{2}=-\left(A u_{1}(t)-A u_{2}(t), w(t)\right) \leq 0
$$

Then

$$
|w(t)|^{2} \leq|w(0)|^{2}=0
$$

Thus $w(t)=u_{1}(t)-u_{2}(t)=0$. And we obtain the uniqueness solution.

Besides $u^{\prime}(t)=-A u(t)$ in $V^{\prime}$, from (1.2) and (1.3), and for all $v \in V$, we have

$$
(A u(t), v) \leq a\|u(t)\|\|v\|
$$

Then $\|A u(t)\|_{V^{\prime}} \leq a\|u(t)\| \quad \forall t \in[0, T]$.

So $A u \in L^{2}\left(0, T, V^{\prime}\right)$ and $u^{\prime} \in L^{2}\left(0, T, V^{\prime}\right)$ 


\section{Regularity and Stability Theorems}

For the regularity proof in two-dimensional case, we will use the following lemma [18]:

Lemma 4. For any open $\Omega$ in $\mathbb{R}^{2}$ and $v \in H_{0}^{1}(\Omega)$ we have

$$
\|v\|_{L^{4}(\Omega)}^{2} \leq \sqrt{2}|v|_{L^{2}(\Omega)}\|v\|_{H_{0}^{1}(\Omega)} .
$$

Theorem 5. Under Hypothesis of the existence theorem 1., the solution u of the problem (1.1) exists in $L^{4}(Q)$ :

$$
u \in L^{4}(Q)
$$

Proof. We know according to the theorem 1. that:

$$
u \in L^{\infty}(0, T, H) \cap L^{2}(0, T, V) .
$$

For all $t \in[0, T]$, we have from the lemma 4 .

$$
\|u(t)\|_{L^{4}(\Omega)}^{2} \leq 2^{1 / 2}|u(t)|\|u(t)\|,
$$

therefore:

$$
\begin{gathered}
\|u(t)\|_{L^{4}(\Omega)}^{2} \leq 2^{1 / 2}|u|_{L^{\infty}(0, T, H)}\|u(t)\| \\
\left(\int_{0}^{T}\|u(t)\|_{L^{4}(\Omega)}^{4} d t\right)^{1 / 4} \leq 2^{1 / 4}|u|_{L^{\infty}(0, T, H)}^{1 / 2}\left(\int_{0}^{T}\|u(t)\|^{2} d t\right)^{1 / 4}
\end{gathered}
$$

hence

$$
\left(\int_{0}^{T}\|u(t)\|_{L^{4}(\Omega)}^{4} d t\right)^{1 / 4} \leq 2^{1 / 4}|u|_{L^{\infty}(0, T, H)}^{1 / 2}\|u\|_{L^{2}(0, T, V)}^{1 / 2}
$$

For the regularity proof in three dimensional case, it sufficient to check that the following lemma [18], is satisfied:

Lemma 6. For any open $\Omega$ in $\mathbb{R}^{3}$ we have

$$
\|u\|_{L^{4}(\Omega)} \leq \sqrt{2}|u|_{L^{2}(\Omega)}^{1 / 4}\|u\|_{H_{0}^{1}(\Omega)}^{3 / 4} \quad \forall u \in H_{0}^{1}(\Omega) .
$$

Theorem 7. Under Hypothesis of the existence of the theorem 1., the solution $u$ of the problem (1.1) verifies:

$$
u \in L^{8 / 3}\left[0, T, L^{4}(\Omega)\right]
$$


Proof. For all $t \in[0, T]$, we have from the lemma 6 .

$$
\|u(t)\|_{L^{4}(\Omega)} \leq \sqrt{2}|u(t)|^{1 / 4}\|u(t)\|^{3 / 4} .
$$

From the theorem 1., $u \in L^{\infty}(0, T, H) \cap L^{2}(0, T, V)$ and we have then:

$$
\|u(t)\|_{L^{4}(\Omega)}^{8 / 3} \leqq 2^{4 / 3}|u(t)|_{L^{\infty}(0, T, H)}^{2 / 3}\|u(t)\|^{2},
$$

and $u \in L^{8 / 3}\left[0, T, L^{4}(\Omega)\right]$.

Theorem 8 (Stability theorem). Let $\mu_{1}$ and $\mu_{2}$ verifie (1) - (5). It is assumed more that

$$
\inf _{s \in \mathbb{R}_{+}} \mu_{1}(s)=b_{1} \neq 0 \text { or } \inf _{s \in \mathbb{R}_{+}} \mu_{2}(s)=b_{2} \neq 0
$$

Then there exists a constant $K>0$ such that

$$
\left|u_{1}-u_{2}\right|_{L^{\infty}(0, T, H)} \leq K \sup _{s \in \mathbb{R}_{+}}\left|\mu_{1}(s)-\mu_{2}(s)\right|
$$

with $K=\frac{1}{\sqrt{b_{1}}}\left\|u_{1}\right\|_{L^{2}(0, T, V)}$ if $b_{1} \neq 0$ or $K=\frac{1}{\sqrt{b_{2}}}\left\|u_{2}\right\|_{L^{2}(0, T, V)}$ if $b_{2} \neq 0$.

Proof. From theorem 1., there exists $u_{1}$ and $u_{2}$ two solutions of the problem (1.1) with $\mu_{1}$ and $\mu_{2}$ (respectively) such that:

$$
\begin{aligned}
& u_{1} \in L^{2}(0, T, V) \cap L^{\infty}(0, T, H) \\
& u_{2} \in L^{2}(0, T, V) \cap L^{\infty}(0, T, H)
\end{aligned}
$$

We take $w=u_{1}-u_{2}$ and $v=w(t)$. We obtain from the equation (1.2):

$$
\frac{1}{2} \frac{d}{d t}|w(t)|^{2}+\int_{\Omega}\left\{\mu_{1}\left[\left|\nabla u_{1}(t)\right|\right] \nabla u_{1}(t)-\mu_{2}\left[\left|\nabla u_{2}(t)\right|\right] \nabla u_{2}(t)\right\} \nabla w(t) d x=0 .
$$

In the case where $\inf _{s \in \mathbb{R}^{+}} \mu_{2}(s) \neq 0$ we add and subtract the term

$$
\int_{\Omega} \mu_{2}\left[\left|\nabla u_{1}(t)\right|\right] \nabla u_{1}(t) \nabla w(t) d x
$$

we obtain

$$
\left\{\begin{aligned}
& \frac{1}{2} \frac{d}{d t}|w(t)|^{2}+\int_{\Omega} \mu_{2}\left[\left|\nabla u_{1}(t)\right|\right] \nabla u_{1}(t) \nabla w(t) d x-\int_{\Omega} \mu_{2}\left[\left|\nabla u_{2}(t)\right|\right] \nabla u_{2}(t) \nabla w(t) d x \\
= & -\int_{\Omega} \mu_{1}\left[\left|\nabla u_{1}(t)\right|\right] \nabla u_{1}(t) \nabla w(t) d x+\int_{\Omega} \mu_{2}\left[\left|\nabla u_{1}(t)\right|\right] \nabla u_{1}(t) \nabla w(t) d x .
\end{aligned}\right.
$$


Then

$$
\left\{\begin{array}{r}
\frac{1}{2} \frac{d}{d t}|w(t)|^{2}+\left(A u_{1}(t)-A u_{2}(t), w(t)\right)= \\
\int_{\Omega}\left\{\mu_{2}\left[\left|\nabla u_{1}(t)\right|\right]-\mu_{1}\left[\left|\nabla u_{1}(t)\right|\right]\right\} \nabla u_{1}(t) \nabla w(t) d x
\end{array}\right.
$$

where $A$ is an operator defined as in (1.3) with $\mu_{2}$.

By using (1.4)

$$
\left(A u_{1}(t)-A u_{2}(t), w(t)\right) \geq b_{2}\|w(t)\|^{2}
$$

we have then:

$$
\frac{1}{2} \frac{d}{d t}|w(t)|^{2}+b_{2}\|w(t)\|^{2} \leq \sup _{s \in \mathbb{R}_{+}}\left|\mu_{1}(s)-\mu_{2}(s)\right|\left\|u_{1}(t)\right\|\|w(t)\| .
$$

It's known, that:

$$
\left(\frac{1}{\sqrt{2 b_{2}}} \sup _{s \in \mathbb{R}_{+}}\left|\mu_{1}(s)-\mu_{2}(s)\right|\left\|u_{1}(t)\right\|-\frac{\sqrt{b_{2}}}{\sqrt{2}}\|w(t)\|\right)^{2} \geq 0,
$$

hence it follows:

$$
\left\{\begin{aligned}
\sup _{s \in \mathbb{R}_{+}}\left|\mu_{1}(s)-\mu_{2}(s)\right|\left\|u_{1}(t)\right\|\|w(t)\| & \leq \frac{1}{2 b_{2}}\left(\sup _{s \in \mathbb{R}_{+}}\left|\mu_{1}(s)-\mu_{2}(s)\right|\right)^{2}\left(\left\|u_{1}(t)\right\|\right)^{2} \\
& +\frac{b_{2}}{2}(\|w(t)\|)^{2}
\end{aligned}\right.
$$

we get then:

$$
\frac{1}{2} \frac{d}{d t}|w(t)|^{2}+\frac{b_{2}}{2}(\|w(t)\|)^{2} \leq \frac{1}{2 b_{2}}\left(\sup _{s \in \mathbb{R}_{+}}\left|\mu_{1}(s)-\mu_{2}(s)\right|\right)^{2}\left(\left\|u_{1}(t)\right\|\right)^{2} .
$$

As

$$
\frac{b_{2}}{2}(\|w(t)\|)^{2}>0
$$

it results then:

$$
\frac{1}{2} \frac{d}{d t}|w(t)|^{2} \leq \frac{1}{2 b_{2}}\left(\sup _{s \in \mathbb{R}_{+}}\left|\mu_{1}(s)-\mu_{2}(s)\right|\right)^{2}\left(\left\|u_{1}(t)\right\|\right)^{2} .
$$

Since $w(0)=0$, we deduce:

$$
\begin{gathered}
|w(t)|^{2} \leq \frac{1}{b_{2}}\left(\sup _{s \in \mathbb{R}_{+}}\left|\mu_{1}(s)-\mu_{2}(s)\right|\right)^{2} \int_{0}^{T}\left\|u_{1}(\tau)\right\|^{2} d \tau \\
|w(t)|^{2} \leq \frac{1}{b_{2}}\left\|u_{1}\right\|_{L^{2}(0, T, V)}^{2}\left(\sup _{s \in \mathbb{R}_{+}}\left|\mu_{1}(s)-\mu_{2}(s)\right|\right)^{2}
\end{gathered}
$$


We have then:

$$
\sup _{t \in[0, T]}\left|u_{1}(t)-u_{2}(t)\right| \leq K \sup _{s \in \mathbb{R}_{+}}\left|\mu_{1}(s)-\mu_{2}(s)\right|
$$

With $K=\frac{1}{\sqrt{b_{2}}}\left\|u_{1}\right\|_{L^{2}(0, T, V)}$.

Therefore

$$
\left|u_{1}-u_{2}\right|_{L^{\infty}(0, T, H)} \leq K \sup _{s \in \mathbb{R}_{+}}\left|\mu_{1}(s)-\mu_{2}(s)\right|
$$

Notice that we can obtain the same result, in the case where $\inf _{s \in \mathbb{R}^{+}} \mu_{1}(s) \neq 0$, if we add and subtract the term

$$
\int_{\Omega} \mu_{1}\left[\left|\nabla u_{2}(t)\right|\right] \nabla u_{2}(t) \nabla w(t) d x .
$$

This completes the proof of the theorem.

Note that all results presented give us sufficient conditions of existence, uniqueness and regularity of the solution. These results also allow us to make a suitable choice data problem considered with a view to approaching numerically.

In this paper we propose a numerical approach by the finite difference methods for the following model:

$$
\left\{\begin{array}{l}
\left.\frac{\partial u}{\partial t}-\operatorname{div}\left(\left(\frac{1}{\sqrt{1+(|\nabla u|)^{2}}}+\alpha\right) \nabla u\right)=0 \quad \text { in } Q=\Omega \mathrm{x}\right] 0, T[ \\
u(x, 0)=u_{0} \quad \text { in } \Omega, \quad u(x, t)=0 \quad \forall x \in \partial \Omega \mathrm{x}[0, T] .
\end{array}\right.
$$

where $u$ represents the processed image.

We consider a bounded domain $\Omega$ in $\mathbb{R}^{2}$, containing strictly the image, in this case we can consider the homogeneous Dirichlet condition on $\partial \Omega$.

\subsection{Discrete PDE's model}

Notice that the problem (4.12) is discretized using the finite differences method. We use an explicit scheme, we denote respectively by $h$ and $k$ the spatial and time steps sizes. In this sequel, we take $h=1$.

For the temporal discretization of the time derivative we have:

$$
\frac{\partial u}{\partial t}(i, j)=\frac{u^{n+1}(i, j)-u^{n}(i, j)}{k}
$$

the superscript $n$ and $n+1$ denote the time levels $t_{n}$ and $t_{n+1}$, respectively. 
For the approximation of the spatial derivatives, a first-order explicit scheme is employed:

$$
\nabla u^{n}(i, j)=\left\{\begin{array}{l}
\frac{\partial u^{n}}{\partial x}(i, j)=\frac{u^{n}(i+1, j)-u^{n}(i, j)}{h} \\
\frac{\partial u^{n}}{\partial y}(i, j)=\frac{u^{n}(i, j+1)-u^{n}(i, j)}{h} .
\end{array}\right.
$$

Then

$$
\left|\nabla u^{n}(i, j)\right|=\sqrt{\left(\frac{u^{n}(i+1, j)-u^{n}(i, j)}{h}\right)^{2}+\left(\frac{u^{n}(i, j+1)-u^{n}(i, j)}{h}\right)^{2}} .
$$

The equations (4.12) can be used as a diffusion problem involving in image processing. We define for every field $p=\left(p_{1}, p_{2}\right) \in \mathbb{R}^{2}$, the discrete divergence approximation:

$$
\begin{aligned}
(\operatorname{div}(p))_{i, j}= & \begin{cases}p_{1}^{n}(i, j)-p_{1}^{n}(i-1, j) & \text { if } 1<i<N_{1} \\
p_{1}^{n}(i, j) & \text { if } i=1 \\
-p_{1}^{n}(i-1, j) & \text { if } i=N_{1}\end{cases} \\
& + \begin{cases}p_{2}^{n}(i, j)-p_{2}^{n}(i, j-1) & \text { if } 1<j<N_{1} \\
p_{2}^{n}(i, j) & \text { if } j=1 \\
-p_{2}^{n}(i, j-1) & \text { if } j=N_{1}\end{cases}
\end{aligned}
$$

where $N_{1}$ is an integer greater than 2 , and

$$
p^{n}(i, j)=\left\{\begin{array}{l}
p_{1}^{n}(i, j)=\left(\frac{1}{\sqrt{1+\left(\left|\nabla u^{n}(i, j)\right|\right)^{2}}}+\alpha\right) \frac{\partial u^{n}}{\partial x}(i, j), \\
p_{2}^{n}(i, j)=\left(\frac{1}{\sqrt{1+\left(\left|\nabla u^{n}(i, j)\right|\right)^{2}}}+\alpha\right) \frac{\partial u^{n}}{\partial y}(i, j)
\end{array}\right.
$$

Then, an iterative method based on the explicit scheme is used to solve the discrete model:

$$
u^{n+1}(i, j)=u^{n}(i, j)+k \operatorname{div}\left(p^{n}(i, j)\right), 1 \leq n \leq M
$$

where

$$
p^{n}(i, j)=\left(p_{1}^{n}(i, j), p_{2}^{n}(i, j)\right)
$$

$u^{n}(i, j)=u\left(x_{i}, y_{j}, t_{n}\right), x_{i}=i h, y_{j}=j h, t_{n}=n k$ and $k=\frac{T}{M}=0.1$ (where $M$ is the number of iterations). 


\section{Numerical Results}

In this section we will present the numerical results obtained by the proposed model. We recall that the SNR is the Signal-to-noise ratio used to estimate the quality of an image $I_{2}$ with respect to a reference image $I_{1}$, defined by the expression:

$$
\operatorname{SNR}\left(I_{1} / I_{2}\right)=10 \log _{10}\left[\frac{\sigma^{2}\left(I_{1}\right)}{\sigma^{2}\left(I_{1}-I_{2}\right)}\right]
$$

where $\sigma$ is the variance.

We begin by presenting a few 2D processed images, obtained by the nonlinear parabolic model with ( $h=1, k=0.1$ and $M$ varies from 50 to 500 ) for processing different medical images corrupted by a Gaussian and Speckle noises, see Figure 1 and 2. We remark that the model gives a better solution for a small values of $\alpha$.

Table (1) is a complete description of the parameter SNR number of experiments that yielded best results for the nonlinear model on images deteriorated by a Gaussian and Speckle noises for $\alpha=10^{-6}$.

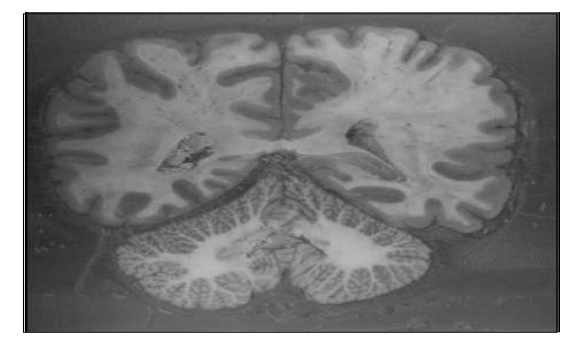

Image original (a)

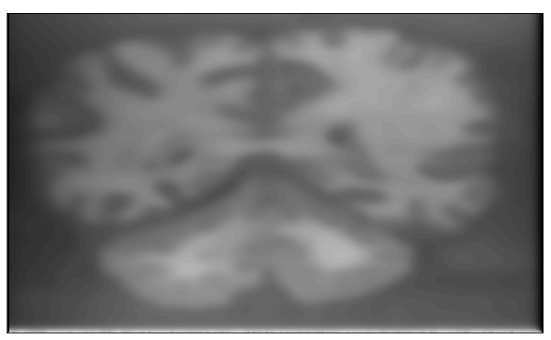

Restored Image with $\alpha=1$

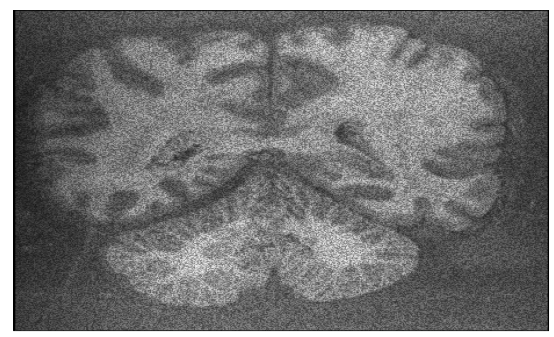

Noisy Image (Speckle)

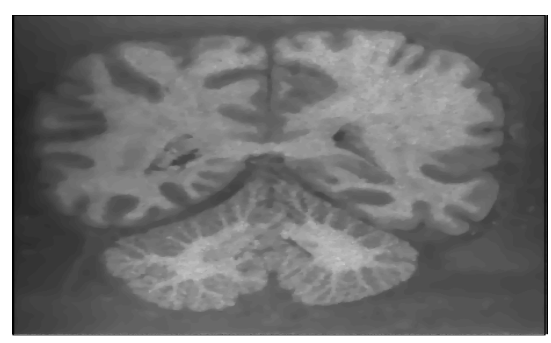

Restored Image with $\alpha=10^{-6}$

Figure 1. Influence of the parametr $\alpha$ on the Restored d'image (Speckle noise) 


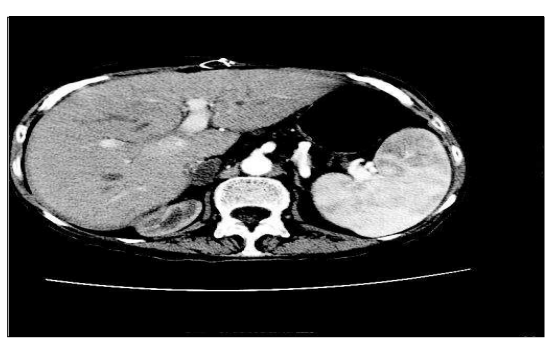

Image originale (b)

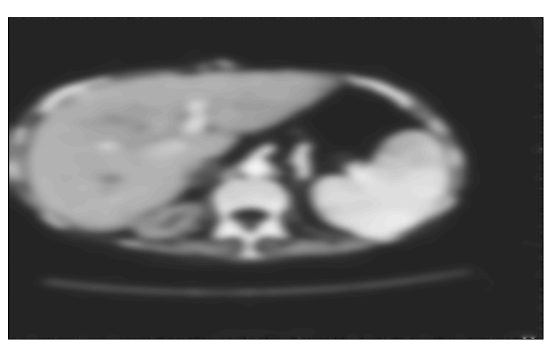

Restored Image with $\alpha=1$

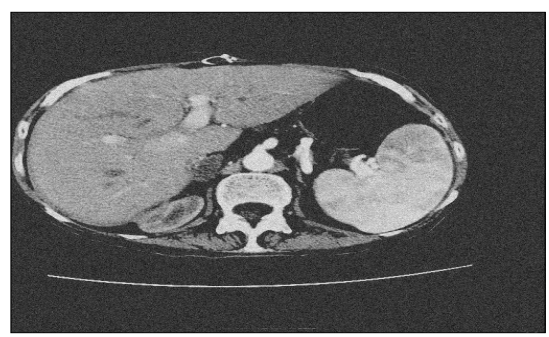

Noisy Image (Gaussian 15\%)

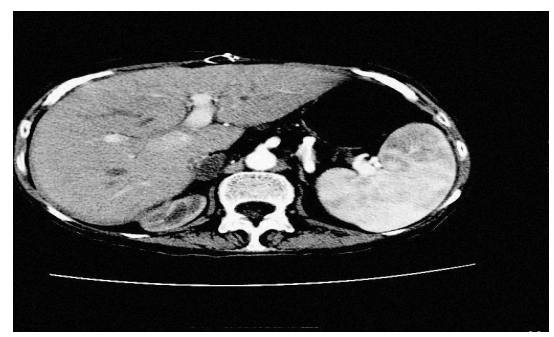

Restored Image with $\alpha=10^{-6}$

Figure 2. Influence of the parametr $\alpha$ on the Restored d'image (Gaussian noise)

\begin{tabular}{|c|c|}
\hline Images & SNR \\
\hline image (a), Speckle Noise & 17.45 \\
\hline image (b), Gaussian Noise 15\% & 26.35 \\
\hline image (b), Gaussian Noise 20\% & 25.93 \\
\hline image (b), Gaussian Noise 30\% & 24.51 \\
\hline
\end{tabular}

Table 1: Evaluation of the noise suppression by the nonlinear restoration method

\section{Conclusion}

We proposed in this work a nonlinear parabolic PDE model for the medical image processing. After the mathematical analysis of the PDE problem, we tested the model numerically. The obtained results show the effectiveness of the approach. 


\section{References}

[1] R. Aboulaich, D. Meskine and A. Souissi, New diffusion models in image processing, Journal of Computers and Mathematics with Applications, (2008), in press.

[2] R. Aboulaich, S. Boujena and E. El Guarmah, Sur un modèle non-linéaire pour le débtruitage de l'image, C.R. Acad. Sci. Paris. Ser. I, 345 (2007), 425-429.

[3] L. Alvarez, P.L. Lions and J.M. Morel, Image selective smoothing and edge detection by nonlinear diffusion, SIAM Journal on Numerical Analysis, 29 (1992), No. 3, 845-866.

[4] G. Aubert and P. Kornprobst, Mathematical problems in image processing, Springer-Verlag, New-York, 2002.

[5] S. Boujena, Etude d'une classe de fluides non newtoniens, les fluides newtoniens généralisés, thèse de 3ème cycle, Université Pierre et Marie Curie -Paris 6, 1986.

[6] F. Catté, P.L. Lions, J.M. Morel and T. Coll, Image selective smoothing and edge detection by nonlinear, SIAM Journal on Numerical Analysis, 29 (1992), 182-193.

[7] A. Chambolle and P.L. Lions, Image recovery via total variation minimization and related problems, Numer. Math. 74 (1997), No. 2, 147-188.

[8] Y. Chen and M. Levine Rao, Variable exponent, linear growth functionals in image restoration, SIAM Journal of Applied Mathematics, 44 (2004), No. 4, 1383-1404.

[9] R. Deriche and O. Faugeras, Les EDP en traitement des images et vision par ordinateur, INRIA research report 2497, 1995.

[10] P. Destuynder, Analyse et traitement des images numériques, Hermès, Paris, 2004.

[11] O. Ladyskenskaya, A. Mathematical problems in the dynamic of viscous incompressible fluid, 2 ème éd. Gordon and Breach, New York, 1969.

[12] S. Levins, Y. Chen and J. Stanich, Image restoration via nonstandard diffusion, TechnicalReport 04-01, Dept of Mathematics and Computer Science, Duquesne University, 2004.

[13] J.L. Lions, Quelques méthodes de résolution des problèmes aux limites non linéaires, Dunod et Gauthier Villard, Paris, 1969.

[14] S. Osher and L. Rudin, Feature-oriented image enhancing using shock filter, SIAM Journal on Numerical Analysis, 27 (1990), No. 4, 919-940.

[15] S. Osher, L. Rudin and E. Fatemi, Non linear total variation based noise removal algorithms, Phys. D, 60 (1992), 259-268.

[16] P. Perona and J. Malik, Scale-espace and edge detection using anisotropic diffusion IEEE Transactions on Pattern Analysis and Machine Intelligence, 12(1990), pp. 429-439. 
[17] L. Rudin, S. Osher and E. Fatemi, Nonlinear total variation based noise removal algorithms, Physica D, 40 (1992), 259-268.

[18] R. Temam, Navier-Stokes equations, Theory and numerical analysis, North Holland, Amsterdam, 1977. 